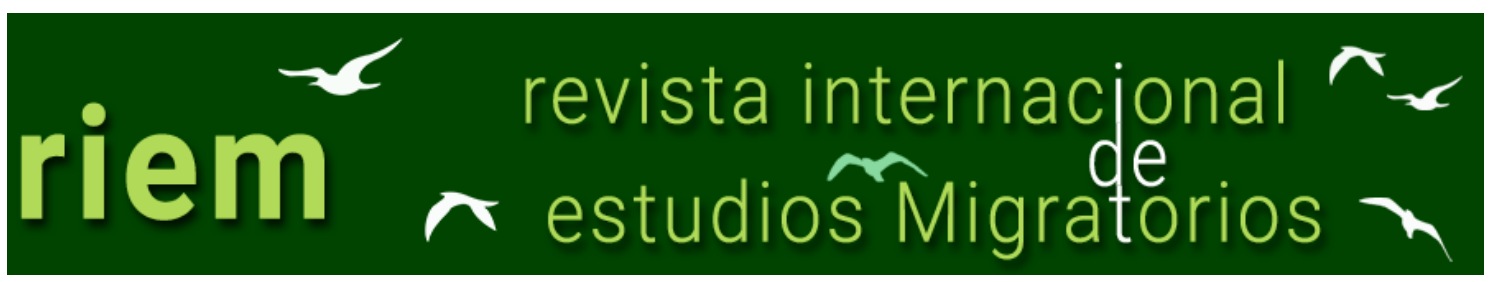

ISSN: $2173-1950$

\title{
Movilidad y migración en el Sistema Científico y Tecnológico en México
}

Eduardo Robles-Belmont ${ }^{1}$

Resumen: La dimensión espacial de las ciencias y las tecnologías es un tema de interés para los estudios sociales de ciencia, tecnología y sociedad. La internacionalización de la ciencia, la fuga de cerebros, las diásporas científicas y las redes de colaboración científica, entre otros, son algunos de los enfoques propuestos en la literatura para abordar la movilidad y la migración de recursos humanos altamente capacitados. Sin embargo, pocos estudios usan datos cuantitativos y visualizan gráficamente la movilidad científica. En este artículo, se analizan datos sobre la movilidad de investigadores durante su formación científica en México y de investigadores que han emigrado hacia este país. Los resultados, bajo la forma de cartografías, caracterizan el perfil de estas movilidades y señalan preguntas sobre la influencia de los centros de atracción científica sobre las agendas y estructuras científicas de los países del Sur, así como sobre sus causas.

Palabras Clave: movilidad científica, migración científica, formación científica, geografía de las ciencias y las tecnologías, México.

\footnotetext{
${ }^{1}$ Laboratorio de Redes, Instituto de Investigaciones en Matemáticas Aplicadas y en Sistemas, Universidad Nacional Autónoma de México, Ciudad de México, México.eduardo.robles@iimas.unam.mx
} 


\title{
Mobility and migration in the Scientific and Technological System in Mexico
}

\begin{abstract}
The spatial dimension of science and technology is a topic of interest for social studies in science, technology and society. The internationalization of science, brain drain, scientific diasporas and networks of scientific collaboration, among others, are some of the approaches proposed in the literature to address the mobility and migration of highly trained human resources. However, few studies use quantitative data and visualize the scientific mobility. In this article, we analyze data on the mobility of researchers during their scientific training in Mexico and of researchers who have migrated to this country. The results, in the form of cartographies, characterize the profile of these mobilities and point out questions about the influence of the centers of scientific attraction on the agendas and scientific structures of the countries of the South, as well as on their causes.
\end{abstract}

Keywords: scientific mobility, scientific migration, scientific training, geography of science and technology, Mexico. 


\section{Introducción}

El quehacer de la producción de indicadores sobre la producción científica y tecnológica ( $\mathrm{CyT})$, desde hace décadas ha ocupado un lugar importante en el ámbito de la política de ciencia y tecnología, así como en los estudios sociales de la ciencia, la tecnología y la sociedad. Existe de hecho una literatura académica bastante amplia donde podemos encontrar trabajos que discuten y proponen nuevas herramientas y metodologías para producir estos indicadores. En las áreas de la Bibliometría y la Cienciometría, así como en otras disciplinas de las Ciencias Sociales (Sociología de la Ciencia, Economía y Gestión), para representar los sistemas científicos y tecnológicos se emplean diversos datos cuantitativos, como el gasto e inversión en ciencia y tecnología, formación de recursos humanos, artículos y patentes publicados, entre otros datos que representen los insumos y los resultados de las actividades científicas y tecnológicas. El uso de este tipo de datos se ha extendido porque permiten caracterizar, monitorear y evaluar la progresión de nuevas ciencias y tecnologías. Por otra parte, el estudio de las nuevas dinámicas en la producción de conocimientos científicos y tecnológicos requiere de indicadores nuevos que reflejen los sistemas estudiados en los nuevos contextos, lo que representa nuevos retos.

Las nuevas dinámicas en la producción, uso y diseminación de conocimientos han sido el objeto de estudios en las ciencias sociales. Los cuales se han interesado en la emergencia de nuevas áreas científicas y tecnológicas, en las redes de colaboración científica, en la acumulación de capacidades, entre otros, como características nuevas de las actividades científicas y tecnológicas. Igualmente, la dimensión espacial es uno de los aspectos que ha llamado la atención de diversos estudios sobre la ciencia y la tecnología. Además, a partir de los enfoques cuantitativos, la dimensión espacial ha sido objeto de varios trabajos académicos que se han centrado en cartografiar el perfil geográfico de la ciencia y la tecnología desde diversas entradas y enfoques (Frenken et al., 2009). En esta literatura se constata la caracterización de diversos aspectos de la dimensión espacial en la producción de nuevos indicadores de las actividades científicas y tecnológicas (Bornmann et al., 2011; Leydesdorff y Persson, 2010). Los resultados de estos estudios son cartografías sobre las colaboraciones científicas y la distribución geográfica de capacidades científicas y tecnológicas.

En la Historia de la Ciencia y la Geografía desde enfoques cualitativos se ha abordado la dimensión espacial de las ciencias durante su producción y circulación de conocimientos (Meusburger et al., 2010). La movilidad y la migración científica y de recursos humanos altamente calificados han sido igualmente estudiadas. El debate en torno a los enfoques teóricos de los estudios de esta movilidad y migración esta aún abierto (Ackers, 2005) y en el marco más amplio de los estudios sobre la migración se 
ha discutido los conceptos teóricos frente a los cambios en los estudios sobre la movilidad y el desarrollo (de Haas, 2010; King, 2012). La movilidad y la migración científica han sido temas de estudio donde la discusión se ha centrado sobre sus aspectos negativos (brain drain y brain waste) y positivos (brain gain)2 ${ }^{2}$ así como sobre otros tópicos que han limitado abordar estos temas por su dimensión y características propias (Delgado-Wise y Chávez-Elorza, 2016). En los países industrializados, el debate sobre la migración de recursos humanos altamente calificados y científicos es aún de actualidad y ha tomado un lugar importante en las políticas públicas de ciencia y tecnología. A partir de diferentes perspectivas, Didou y Gérard (2009) han coordinado la publicación de diversos trabajos cuyos aportes ayudan a dar cuenta de las dinámicas de la movilidad científica y de recursos humanos altamente calificados en América Latina y el Caribe. No obstante, Didou (2009) señala que los estudios sobre la movilidad científica son aún insuficientes y que uno de los límites de éstos estudios reposa sobre la diversidad y la inconsistencia de los indicadores. Este límite no es menor, ya que impide poder efectuar estudios comparativos sobre este tipo de movilidad y migración en la región. Por ejemplo, para el caso de México, Gérard y Maldonado (2009) se han limitado a analizar comunidades académicas adscritas a una sola universidad. Estos trabajos no son negligentes, ya que aportan elementos para comprender estos fenómenos sociales, pero a partir de estos estudios es difícil concluir tendencias nacionales y efectuar una caracterización más representativa de las dinámicas de la movilidad y migración científicas en este país.

Este trabajo se centra sobre la movilidad de investigadores en México durante su formación doctoral fuera de este país. El objetivo central es caracterizar la movilidad de los investigadores mexicanos durante sus estancias en el extranjero en su formación científica, así como caracterizar la migración científica instalada en México a través de la movilidad de científicos que se han instalado en este país. Los datos de este estudio conciernen a los investigadores miembros del Sistema Nacional de Investigadores (SNI) en el año 2013. Estos datos nos permiten identificar los lugares de formación doctoral y posteriormente los lugares de trabajo de estos investigadores. Además, es posible cruzar datos para conocer las disciplinas del conocimiento de formación científica y conocer la evolución de este tipo de movilidad de recursos humanos

\footnotetext{
2 En los estudios sobre migración calificada y científica existen diversos términos en la literatura usados para designar el tipo de migración y describir las implicaciones positivas o negativas al desarrollo de los páises de origen o destino. El termino brain drain define la migración calificada que representa la perdida de recursos humanos para el desarrollo de los países de origen (Docquier et al., 2005) y el término brain gain indica cuando esta migración genera beneficios al ejercer su profesión y conocimiento tanto para el país de acojo como para el país de origen (Freitas et al., 2012). Por otro lado, el término brain waste ha sido propuesto para indicar cuando el migrante calificado parte de su país y no ejerce su formación profesional o sus habilidades y conocimientos adquiridos en el país de origen o de destino (García Pires, 2015; Mattoo et al., 2005).
} 
altamente calificados. Con el apoyo del análisis estadístico y del análisis de redes sociales aplicados a la localización geográfica de los lugares de formación doctoral, los resultados presentados en este estudio constituyen un panorama de esta movilidad y de la migración científicas en México.

Este texto se compone de cuatro partes, la primera parte presenta una breve revisión de la literatura sobre la dimensión espacial en los estudios sociales de ciencia y tecnología. Enseguida, se detalla la metodología empleada en el estudio. Posteriormente, los resultados son expuestos centrándose en visualizaciones de cartografías y finalmente las conclusiones se exponen.

\section{Dimensión espacial, circulación de conocimientos, movilidad y geografías}

La dimensión espacial no es un tema nuevo en los estudios sociales de ciencia, tecnología y sociedad. Desde hace al menos dos décadas, estudios interdisciplinarios se han interesado a la cartografía de la ciencia. En particular, estudios en Historia de la Ciencia y en Geografía están de acuerdo sobre la idea de que la ciencia posee una geografía y que tiene huellas del contexto particular donde es producida (Jöns et al., 2010). Además, la Historia de la Ciencia nos ha mostrado que los conocimientos científicos responden a las visiones e intereses sociales ligados a los espacios de producción y de su uso (Livingstone, 2003; Mayer, 2015). La dimensión espacial es igualmente interesante de considerar en el análisis de nuevas ciencias y tecnologías ya que nos permite observar cómo las trayectorias socio-economicas de las regiones juegan roles importantes en la instalación y la orientación de insfraestructuras tecnológicas (Luna-Ochoa et al., 2016). Abordar la dimensión espacial de los conocimientos científicos y tecnológicos implica revisar y discutir enfoques y conceptos donde los lugares y los contextos de producción de conocimientos, así como su circulación, son presentes. Sobre este último punto, Quet (2014) señala que la circulación de conocimientos ha tomado un lugar importante en las ciencias sociales y se basa sobre tres ejes: los elementos de la circulación, la movilidad y la naturaleza de los conocimientos. En efecto, la noción de "circulación” surge en los estudios centrados sobre la dimensión espacial ya que observamos los desplazamientos de los diversos elementos (individuos, objetos, construcciones simbólicas, entre otros) en los procesos de producción de conocimientos. Los desplazamientos de estos elementos no tienen fronteras, y las trayectorias y contextos del uso que se les da pueden influir sobre la expansión y consolidación de las disciplinas (Mayer, 2015). Por otra parte, otro tema sobre la movilidad es la transferencia de conocimientos, ya que es a través de la 
movilidad de estudiantes, de técnicos y de investigadores que los conocimientos y las prácticas científicas se diseminan de un lugar a otro. Seguir los elementos en circulación es entonces interesante, esto sin dejar de lado la observación de la manera cómo estos elementos se movilizan y forman parte de nuevos conocimientos en otros contextos. Además, al estudiar la movilidad de los investigadores durante su formación nos aproximamos a los estudios de la migración internacional de estudiantes. Este tipo de migración es un fenómeno global con implicaciones nacionales y regionales, cuyos estudios no son suficientes para entenderlo y aún hay un debate sobre el marco teórico y conceptual para el abordaje de este tipo de migración (Findlay et al., 2012; Freitas et al., 2012; King y Raghuram, 2013; Raghuram, 2013).

El análisis de la circulación de conocimientos puede entonces ser muy amplio y el marco analítico tomar forma a partir de los ejes evocados por Quet. Al revisar la literatura académica, se puede constatar las diferentes preguntas en torno a los diferentes estudios sobre la movilidad y migración científicas. En los países industrializados, las preguntas se centran por ejemplo sobre los aportes de la migración calificada al mercado laboral y la economía (Straubhaar, 2000), así como sobre la carrera para la atracción de talentos (Docquier y Machado, 2016; González Ramos, 2011), entre otros, mientras que en los países emergentes o no industrializados las preguntas se interesan sobre la fuga de cerebros y las posibles ventajas de las diásporas científicas (Delgado-Wise y Chávez-Elorza, 2016; Didou, 2009; Meyer et al., 2001; Pellegrino, 2001; Tejada, 2012). En todos los casos, estos estudios contribuyen con elementos para la política pública de ciencia y tecnología, así como para el desarrollo, aunque la relación migración-desarrollo tiene una concepción unilateral y distorsionada (Delgado-Wise et al., 2009).

En América Latina, estos temas han sido abordados desde el enfoque de la internacionalización de la ciencia (Vessuri, 2003), la fuga de cerebros (Didou y Gérard, 2009; Özden, 2006), las diásporas científicas (Meyer y Brown, 1999) y las redes de colaboración científica (Contreras-Gómez et al., 2015; Robles-Belmont, 2014). En los estudios de la innovación, la dimensión espacial ha sido igualmente presente en el marco del enfoque de los Sistemas Regionales de Innovación (Valdez-Lafarga y LeónBalderrama, 2015). Estos estudios tienen en común considerar a los recursos humanos altamente calificados como objetos de estudio, de los cuales la movilidad se exacerba con la globalización y en algunos casos las políticas públicas son igualmente parte de los análisis. Además, la mayoría de los trabajos son cualitativos y la poca disponibilidad de datos sobre la movilidad de este tipo de recursos humanos dificulta la realización de estudios precisos y comparativos (Didou, 2009; Özden, 2006). 
En lo que concierne a México, diversos estudios sobre la movilidad científica han sido efectuados desde los enfoques brain drain y brain gain. Frente a las dificultades para el acceso y el tratamiento de datos sobre la movilidad y la migración científica, así como de otros recursos humanos altamente calificados, pocos son los estudios que proponen análisis cuantitativos y que nos permiten tener panoramas amplios sobre esta movilidad en México. Las preguntas de los estudios realizados sobre México se han centrado sobre las trayectorias de los investigadores formados en el extranjero y las repercusiones sobre las estructuras científicas del país (Gérard y Maldonado, 2009), las correlaciones entre los lugares de formación, las redes de colaboración y la productividad científica (Gérard y Kuri, 2009) y las características de la movilidad en el campo de la física (Contreras-Gómez et al., 2015), entre otros. El abordaje de estos temas y los resultados de estos estudios aportan en efecto al estudio de la movilidad, sus orígenes e implicaciones. Sin embargo, estos estudios se caracterizan por centrarse sobre estudios de caso particulares de universidades o disciplinas, lo que conlleva límites para obtener conclusiones más amplias en torno a la movilidad científica en México e identificar tendencias a nivel nacional, así como poder efectuar análisis por campos del conocimiento, regiones, estatus y género de los investigadores, entre otros elementos de análisis.

Como ya se mencionó líneas arriba, en este artículo se expone un panorama de la movilidad y la migración científicas en México a partir de la visualización de datos con el fin de obtener representaciones de los flujos de los investigadores que forman parte de la estructura científica y tecnológica de este país. Estas representaciones visuales son expuestas bajo la forma de cartografias donde la distribución geográfica de los desplazamientos de los actores científicos puede ser visualizada desde diferentes entradas de datos. En este contexto, exponemos dos cartografías. La primera expone la movilidad de los investigadores mexicanos que han efectuado estudios de doctorado en el extranjero, lo que permite identificar los "polos de atracción científicos" en términos de países. La segunda cartografía expuesta representa la migración científica hacia México, la cual no es negligente y constata la importancia de la internacionalización de la ciencia en este país.

\section{Metodología}

Líneas arriba se evocó que la disponibilidad de datos sobre la movilidad y la migración científica han sido obstáculos para efectuar estudios cuantitativos y representativos de los sistemas científicos y tecnológicos. En el caso mexicano, el Consejo Nacional de Ciencia y Tecnología (CONACyT) es el organismo público 
encargado de elaborar las políticas de ciencia y tecnología, de crear los mecanismos necesarios para la aplicación de las políticas, de gestionar recursos públicos para el desarrollo de ciencias y tecnologías, así como de fungir como el principal intelocutor entre los diferentes organismos internacionales para la cooperación científica y tecnológica. En este marco, el SNI está bajo la tutela del CONACyT. Dentro de sus funciones como organismo público y después de la Ley Federal de Transparencia y Acceso a la Información Pública del año 2003, el CONACyT cuenta con una plataforma para permitir el acceso a la información pública bajo la supervisión del Instituto Nacional de Transparencia, Acceso a la Información y Protección de Datos Personales. Es entonces gracias a las reformas para garantizar los derechos al acceso a la información pública y al desarrollo de las tecnologías de la información y de la comunicación que existen recientemente posibilidades de acceso a datos útiles para efectuar estudios más amplios sobre la movilidad científica (Delgado-Wise y ChávezElorza, 2016).

Los datos empleados en este estudio han sido obtenidos a través de una solicitud de acceso a información pública en la plataforma mencionada en el párrafo anterior. Con esta información se construyó una base de datos que concierne a 19,740 investigadores miembros del SNI en el año 2013. Este número de investigadores no es la totalidad de los científicos mexicanos, sin embargo, el SNI aglomera a los científicos con mayor productividad en términos de publicaciones, formación de recursos humanos y otras actividades académicas en las diversas disciplinas que se cultivan en todas las entidades académicas del país, por lo cual considerarlo como objeto de estudio es representativo del sistema científico mexicano. Para poder identificar a los investigadores que han efectuado sus estudios doctorales en el extranjero se empleó el campo de la base de datos que indica el país de formación doctoral de los miembros del SNI. Por otro lado, para los investigadores que nacieron fuera de México, se utilizó el campo que indica el país de nacimiento. Aquí es importante señalar que la utilización de estos datos ha sido posible gracias a la estructura de la base de datos para su consulta y también poder cruzar la información con otras bases de datos. A pesar de contar con una base de datos bien estructurada, ha sido necesario revisar la información y complementar los datos concernientes a 100 investigadores, ya que la información proporcionada por el CONACyT contenía campos vacíos y errores de captura. La manipulación de los datos ha consistido en efectuar análisis estadístico descriptivo y construir matrices de adyacencia a fin de poder visualizar los datos bajo la forma de redes que representen la movilidad de los investigadores.

Para producir las cartografías y trazar geográficamente las trayectorias de los investigadores desde su país de nacimiento o el país donde han realizado sus estudios 
doctorales, se usó el programa informático para el análisis de redes sociales Pajek y el método propuesto por Leydesdorff y Persson (2010). Este método consiste en remplazar las coordenadas de la distribución de los nodos en la estructura de los archivos para el análisis de redes sociales (archivos .net) por la latitud y la longitud de los países. La geocodificación de los datos se realizó con la aplicación en línea de GPS Visualizer (http://www.gpsvisualizer.com/geocoder/). Finalmente, la visualización de la red que representa la movilidad de los investigadores ha sido sobrepuesta sobre un mapamundi.

Aquí es pertinente de señalar que el interés de usar estas cartografías reposa en la búsqueda de generar representaciones visuales de la movilidad científica. Las cartografías son interesantes de usar en estos estudios ya que nos permiten obtener y utilizar fácilmente informaciones sobre una realidad compleja. El uso de cartografías permite omitir detalles innecesarios y centrar la atención de los lectores sobre la información y las preguntas claves, así como comunicar ideas de una manera muy comprensible a no especialistas (Miller y Page, 2007). El uso de las cartografías para los estudios sobre la movilidad y la migración potencializa en efecto las capaciades explicativas y, además, con las herramientas informáticas actuales su reproducción es una tarea sencilla.

\section{Perfil de la movilidad de la formación de los científicos mexicanos}

Antes de continuar con los resultados de este estudio, es pertinente dedicar algunas líneas al SNI con el fin de contextualizar la fuente de los datos empleados, así como la representación del sistema científico y tecnológico mexicano. El SNI de México ha sido creado en 1984 como una iniciativa para el estíomulo económico de los investigadores. Los miembros de este sistema son en efecto beneficiados de una atribución económica mensual basada en su productividad académica. Actualmente, el SNI ha pasado de una dimensión económica a una dimensión simbólica, ya que ha jerarquizado a la elite científica mexicana (Didou y Gérard, 2010). Los requisitos para pertenecer, mantenerse y promoverse en el SNI son bastante exigentes: es necesario contar con el título de doctorado, publicar en revistas indizadas, no tener más de 40 años para ingresar y laborar en una institución universitaria o de investigación. Desde 1984, el número de investigadores que conforman el SNI no ha dejado de crecer, según las estadísticas del CONACyT. De hecho, su crecimiento anual en promedio es del 11\%. Claro está que el SNI no es la totalidad de los investigadores mexicanos, pero considerando el número de investigadores que lo conforman puede ser considerado como representativo de la comunidad científica mexicana. 
En el año 2013, un total de 19740 investigadores conforman el SNI, de los cuales el 34.86\% han efectuado su formación doctoral en el extranjero, sea un total de 6882 investigadores. Por una parte, esta cifra confirma la importancia de la movilidad de los recursos humanos en la formación de las comunidades científicas en México. Por otra parte, este porcentaje hace emerger preguntas sobre las implicaciones que puede tener esta movilidad en las estructuras y prácticas de estas comunidades (Gérard y Maldonado, 2009).

La movilidad científica no es un fenómeno nuevo, en la historia de la ciencia y de los conocimientos científicos se han publicado diversos trabajos sobre la movilidad de individuos, teorías, textos, ideas, instrumentos científicos, entre otros (Livingstone, 2003). La movilidad científica ha tenido diferentes etapas en la historia de la humanidad y se encuentra en medio de preguntas sobre la fuga de cerebros, la transferencia de conocimientos y la conformación de comunidades científicas internacionales (Ziman, 1977). Además, la movilidad científica ha aumentado con la globalización y la internacionalización de las universidades (Vessuri, 2003).

En la curva de la Figura 1 se muestra como esta internacionalización de la ciencia ha evolucionado en México a través de la movilidad de los investigadores mexicanos que han efectuado sus estudios de doctorado en el extranjero. El crecimiento de la movilidad que se muestra en esta figura durante las últimas dos décadas refleja en efecto el resultado de la internacionalización de la ciencia que ha sucecido con la emergencia de nuevas ciencias y tecnologías: Tecnologías de la Información y la Comunicación, Biotecnologías, Nanotecnologías, entre otras. Esta movilidad creciente puede igualmente explicarse con los importantes recursos económicos destinados por el CONACyT y otras entidades públicas mexicanas para efectuar estudios en el extranjero. En el año 2004, del total de las becas financiadas por el CONACyT el 16.51\% fueron para efectuar estudios de posgrado en el extranjero. Por otro lado, es interesante resaltar la caída en la curva en el periodo analizado, ya que esto sugiere que la inserción de los investigadores en el SNI es de alrededor de 3 años. 
Figura 1. Evolución de la obtención del doctorado en el extranjero de investigadores miembros del SNI hasta el año 2013

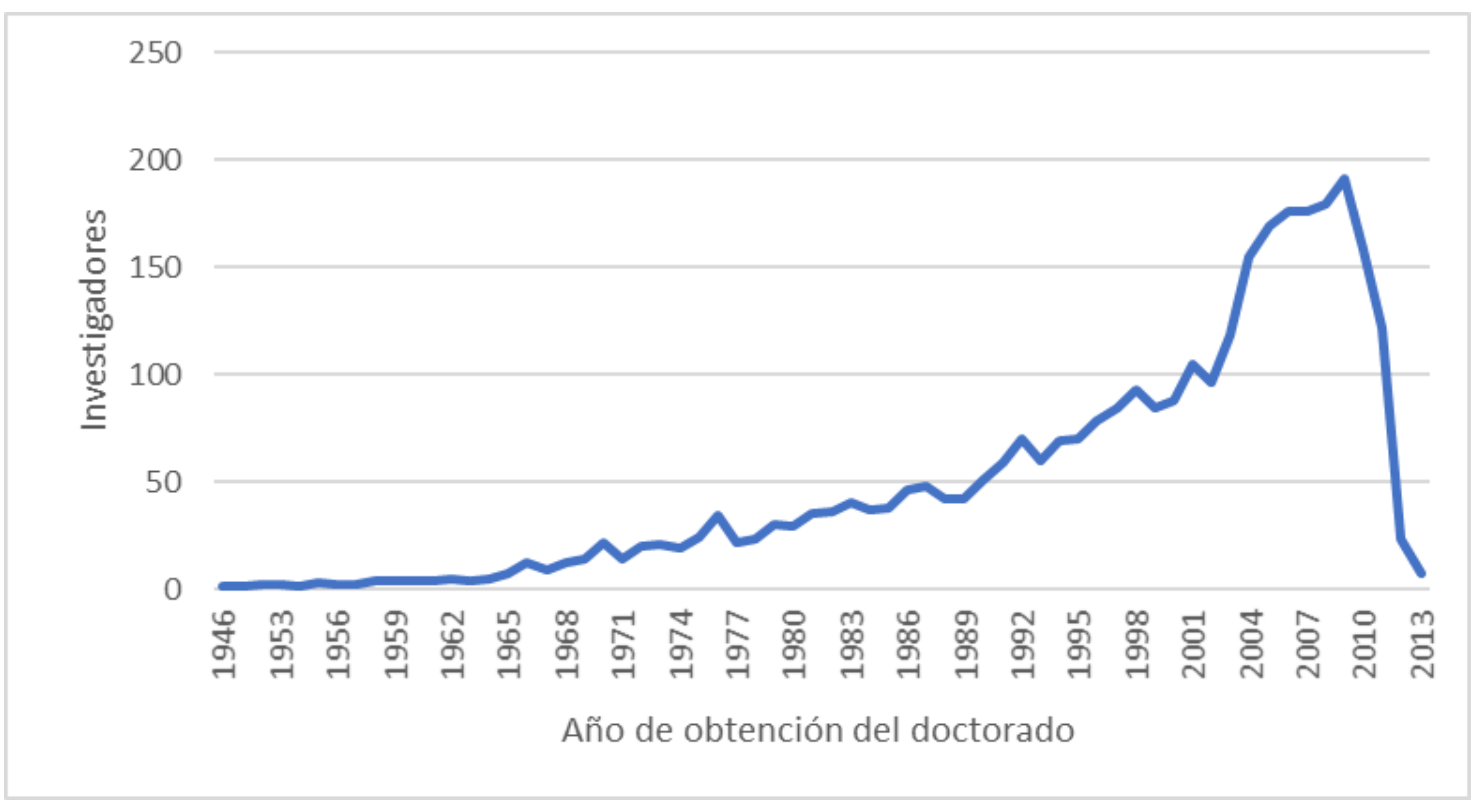

Fuente: Elaboración propia con datos del SNI 2013

En el SNI la investigación está organizada en 7 áreas y posteriormente en 26 campos del conocimiento. Observar la distribución de los investigadores en los campos y áreas del conocimiento permite identificar la distribución de la movilidad en las categorías de la ciencia y la tecnología en México. En la Figura 2 se expone una visualización basada en el análisis de redes sociales que muestra las relaciones entre los 7 campos y las 26 áreas del conocimiento, según como está organizada la investigación en el SNI. Esta visualización muestra que el campo más representativo es el de la Físicomatemáticas y ciencias de la tierra, en el cual un total de 1371 investigadores efectuaron sus estudios de doctorado en el extranjero. Este campo está distribuido sobre 8 áreas del conocimiento, entre las que sobresalen la Física (534 investigadores), Ciencias de la Tierra y del Cosmos (419 investigadores) y Matemáticas (366 investigadores). Enseguida, el segundo campo más representativos es la Ciencia de la Tecnología (1226 investigadores) y las Ciencias Sociales (1221 investigadores). Estos resultados constatan, por una parte, la importancia de los campos científicos y tecnológicos en el sistema científico mexicano, importancia que ya ha sido señalada en otros estudios (Contreras-Gómez et al., 2015; Robles-Belmont et al., 2013). Por otra parte, esta distribución de la movilidad en los campos y áreas del conocimiento muestra el rol central que ha jugado la movilidad científica internacional en la formación de comunidades científicas en México.

Hasta aquí nos hemos centrado sobre la caracterización del crecimiento y de la distribución de la movilidad en las diferentes áreas y campos del conocimiento. 
Visualizar la distribución geográfica de la movilidad científica es el objetivo central de este estudio, se trata de representar la movilidad de los investigadores mexicanos durante sus estudios de doctorado y sus lugares de trabajo académico. En la cartografía de la Figura 3, se muestra esta movilidad distribuida sobre un total de 47 países, incluido México. Las flechas indican los desplazamientos entre México y los países donde los investigadores han efectuado sus estudios doctorales. Solamente para los 4 países más representativos se muestran las cifras de la movilidad, esto con el fin que la cartografía sea legible. La diferencia de la movilidad de partida y de retorno en estos casos refleja una parte de la fuga de cerebros (brain drain). Además, esta representación de la movilidad de los investigadores mexicanos muestra la configuración internacional de los polos de atracción científica, de los cuales para las comunidades científicas mexicanas los polos atractivos son los países de Europa Occidental y los Estados Unidos.

Por otra parte, la movilidad científica mostrada en la Figura 3 muestra los desplazamientos de los investigadores al final de su formación doctoral y se puede observar la movilidad de los investigadores mexicanos que no han retornado. Esta movilidad, que es en efecto parte de la fuga de cerebros y de la diáspora científica, representa igualmente una parte de la distribución del mercado del trabajo científico a nivel internacional. Entonces, es interesante observar esta distribución espacial y cognitiva (en términos de áreas y campos del conocimiento) con el fin de tener panoramas de la organización del trabajo científico a nivel internacional. Otra posible lectura de esta visualización de la movilidad es la asimetría de la distribución de las capacidades científicas y tecnológicas, esto no solamente en términos de recursos humanos altamente capacitados, sino que en términos de la disponibilidad de insfraestructuras científicas y tecnológicas. De hecho, en otros estudios se ha señalado el papel de la infraestructura en la organización del trabajo científico y tecnológico en el caso de las tecnologías emergentes (Robles-Belmont, 2009). El acceso a las infraestructuras científicas en uno de los elementos tomados en cuenta en las elecciones de los jóvenes investigadores para efectuar sus extudios en el extranjero. 


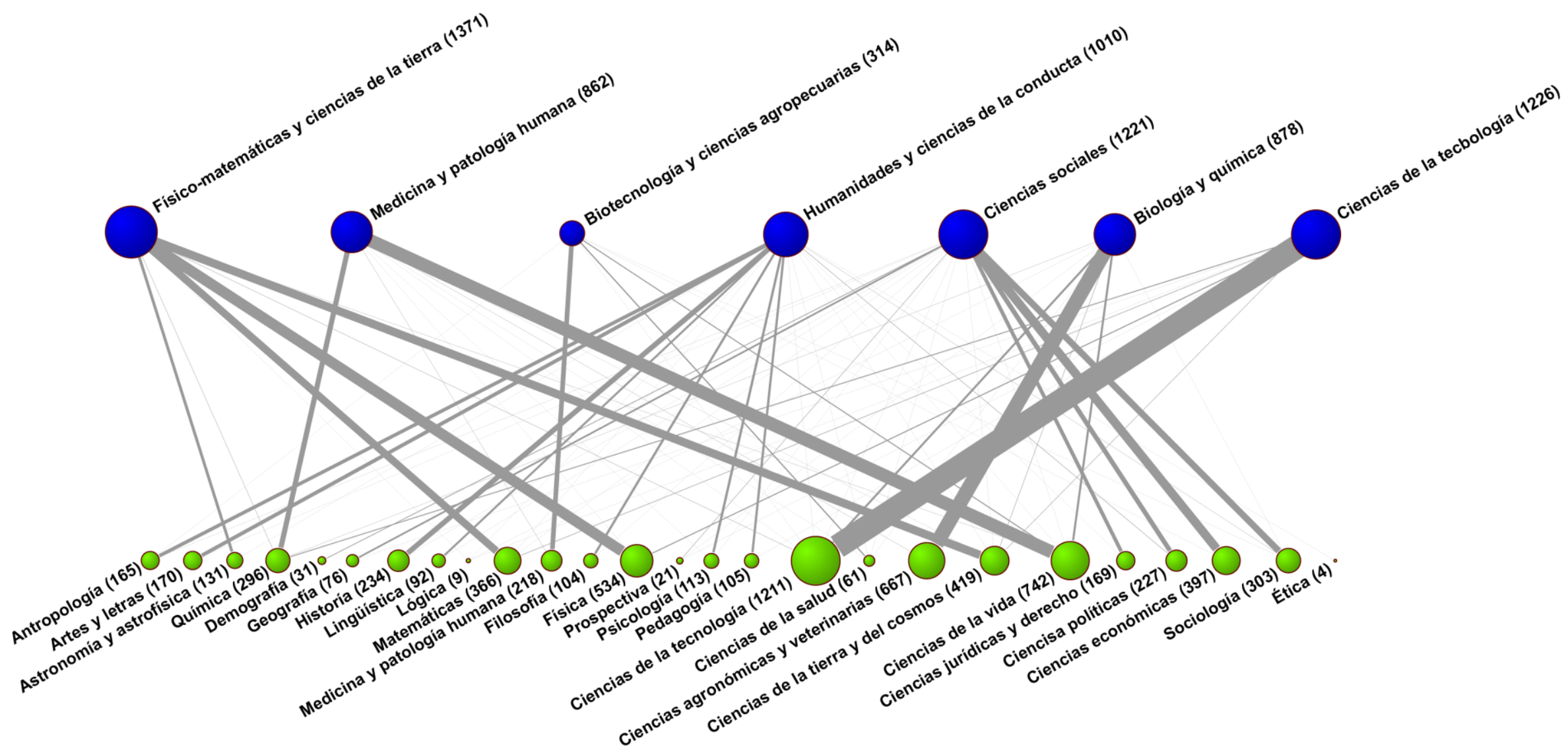

Figura 2. Áreas y campos científicos y tecnológicos en la movilidad de investigadores miembros del SNI en México que han efectuado sus estudios de doctorado en el extranjero

Fuente: Elaboración propia con datos del SNI 2013 


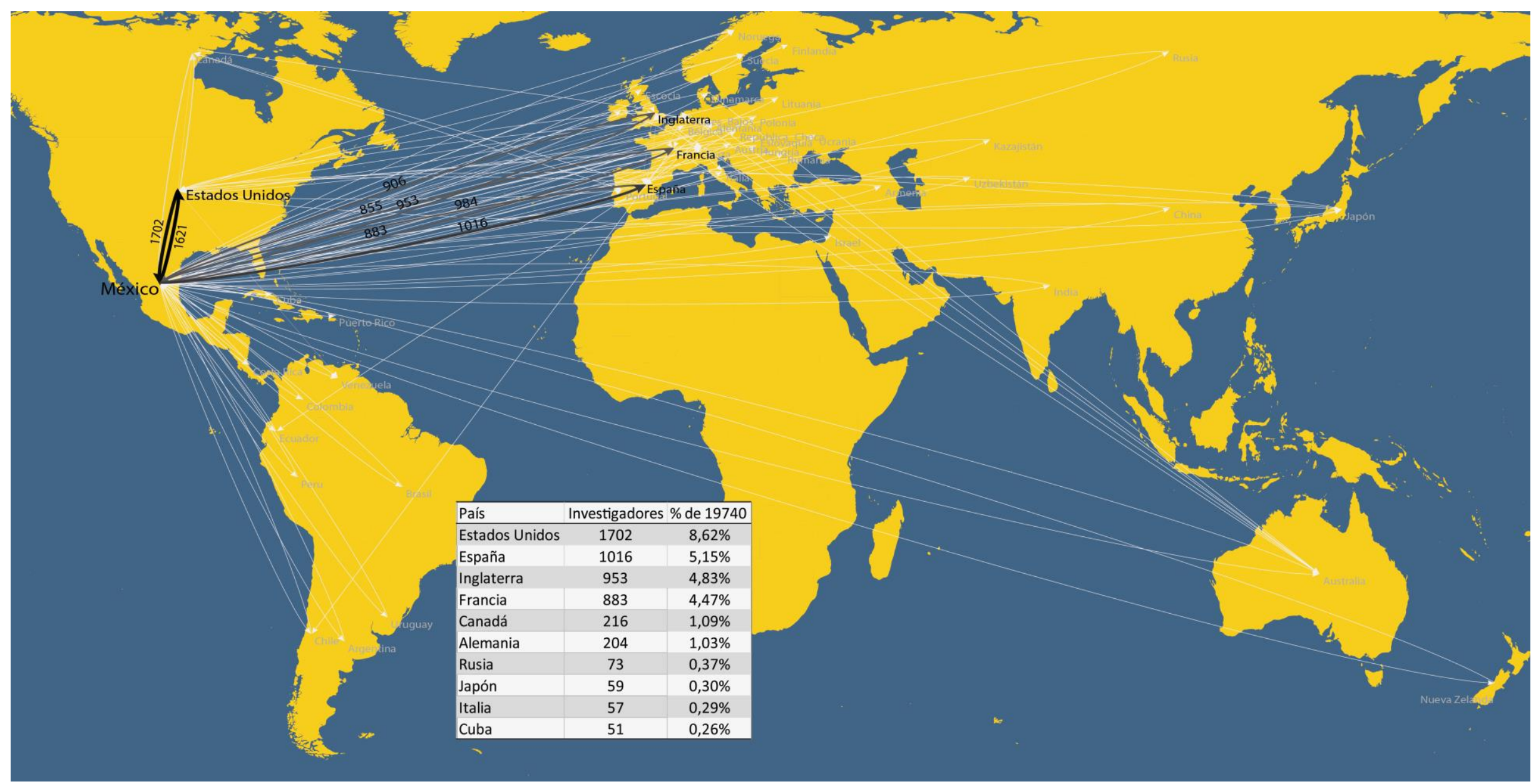

Figura 3. Cartografía de la movilidad durante la formación doctoral de los investigadores científicos mexicanos miembros del SNI hasta el año 2013

Fuente: Elaboración propia con datos del SNI 2013 
En lo que concierne a la migración científica, en la primera cartografía podemos observar la movilidad de una parte de esta migración mexicana vista como fuga de cerebros y parte de la diáspora científica. En México, estos dos temas han sido objeto de diversos trabajos académicos, así como de políticas y acciones de instituciones gubernamentales que buscan disminuir la fuga de cerrebros y obtener algún beneficio de las diásporas científicas. Un ejemplo es el programa de Cátedras para Jóvenes Científicos lanzada por el CONACyT en el año 2014, iniciativa que tiene como objetivo reforzar las capacidades humanas y materiales del sistema científico y tecnológico. Con anterioridad a esta iniciativa, otros esfuerzos han visto la luz, tales como las Redes de Talentos Mexicanos en el Extranjero y el Programa de Repatriamiento y Retención de investigadores mexicanos, lanzado en el año 2006. Sin embargo, a pesar de estas iniciativas que no son negligentes, la migración de investigadores mexicanos persiste.

Otro dato sobre el SNI que es interesante de observar concierne a los investigadores nacidos fuera de México y que laboran en universidades y centros de investigación en este país, se trata en este caso de la migración científica hacia México. Para el año 2013, un total de 1927 investigadores nacidos fuera de este país son parte del SNI (el 9.76\% del total de este sistema), cifra que es tan importante como la de los investigadores mexicanos formados en el extranjero. En la Figura 4, se muestra la cartografía de esta movilidad científica hacia México, cuyo panorama no es el mismo que el obtenido en la primera cartografía. La distribución de la movilidad es diferente ya que se aprecia en efecto una movilidad importante de países del Norte hacia México, pero igualmente se observa una movilidad que involucra a países que no figuran en la primera cartografía. Tambien es interesante observar de cerca esta cartografía ya que ilustra la trayectoria de estos investigadores antes de llegar a México, lo que hace emerger preguntas sobre las causas de este tipo de migración. Para abordar estas cuestiones, el estudio de las trayectorias de los investigadores y de los contextos de los orígenes puede aportar elementos centrales para describir y comprender estos fenómenos. 


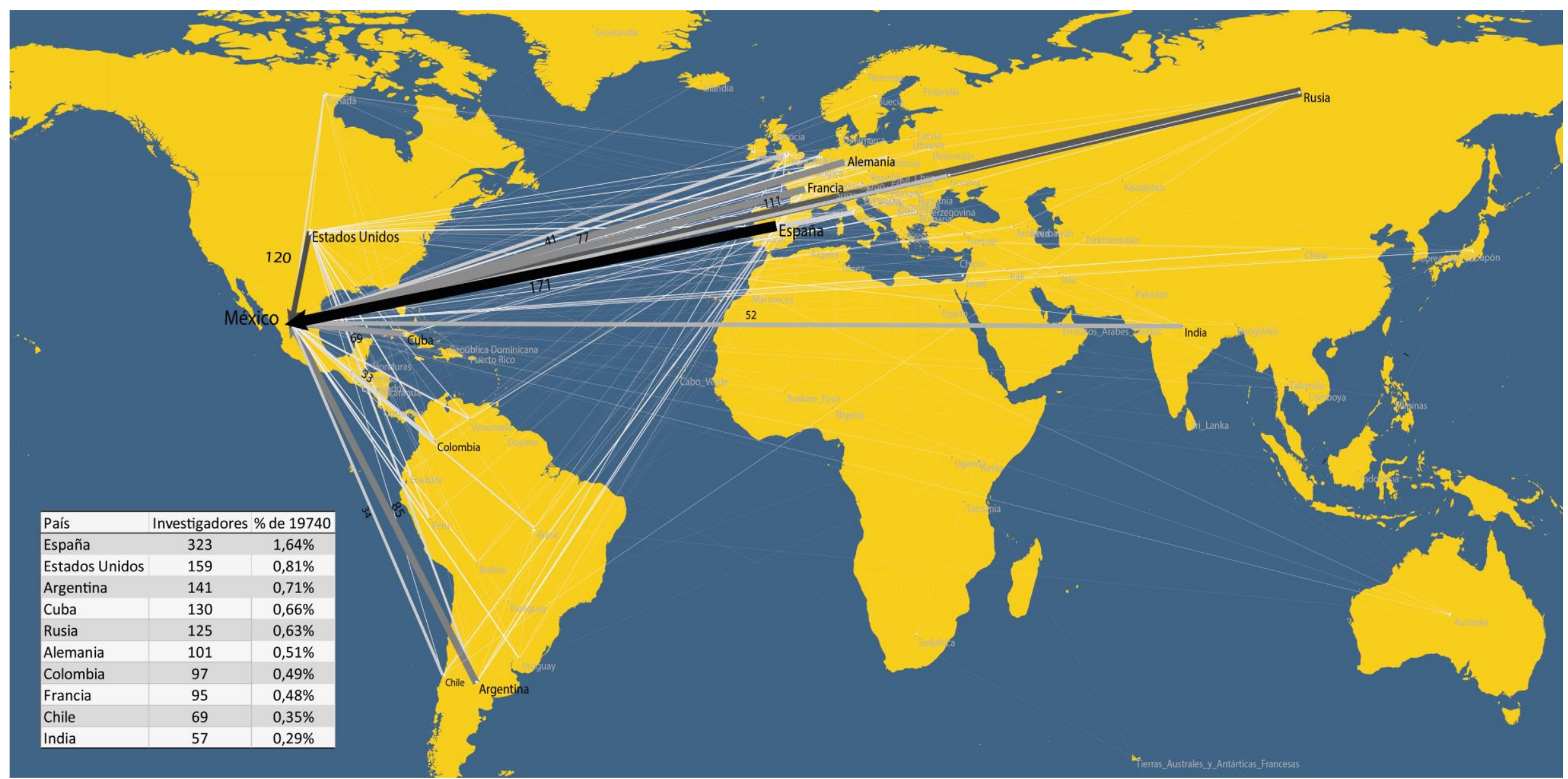

Figura 4. Cartografía de la movilidad de los investigadores miembros del SNI hasta el año 2013 nacidos fuera de México Fuente: Elaboración propia con datos del SNI 2013 
En este texto se han evocado algunos puntos interesantes para explorar con este tipo de visualizaciones sobre la movilidad de la migración científica en México, siendo entonces su caracterización expuesta en este trabajo el aporte central de este artículo. Sin embargo, estos resultados dicen muy poco sobre las causas de estas movilidades, las cuales, como se muestra en este estudio, son importantes para el sistema científico mexicano. Otros estudios sobre la movilidad y la migración científicas en otros países y regiones han señalado un amplio abanico de posibles causas (Ackers, 2005). Causas que van desde las elecciones personales hasta conflictos políticos y sociales que desplazan poblaciones. Además, las elecciones de los jóvenes investigadores para efectuar sus estudios o trabajar en instituciones científicas son determinadas por diversos factores como el idioma del país anfitrión, la visibilidad y el prestigio de las instituciones y comunidades científicas, las políticas locales en temas de migración, el mercado laboral, entre otros.

\section{Conclusiones}

En este texto se expusieron resultados de la caracterización de la movilidad de científicos mexicanos durante su formación doctoral. Los resultados son las cartografías sobre la movilidad y la migración científica en México. Los datos sobre la movilidad que se analizan conciernen a un total de 6882 investigadores inscritos en el Sistema Nacional de Investigadores en el año 2013 y los datos para la migración hacia este país conciernen a 1927 investigadores nacidos fuera de México. Ambas cifras muestran que la internacionalización de la ciencia y la tecnología juega, en efecto, un papel importante en el sistema científico de este país. Además, esta movilidad vista a partir de las áreas y campos del conocimiento revela diferencias marcadas en la formación de capacidades cientificas, de las cuales surge la pregunta sobre la influencia que los centros de atracción científica pueden tener sobre las agendas de la investigación de las instituciones mexicanas. Observar de cerca esta movilidad puede en efecto darnos pistas sobre hasta que punto la movilidad puede tener el rol de agente en la expansión científica (Mahroum, 2000). Es evidente que para abordar estas preguntas es necesario un estudio y que además se nutra de trabajos previos que se han cuestionado estos temas sobre la construcción de las agendas científicas (Kreimer, 2006; Kreimer y Zabala, 2006).

Las dos cartografías obtenidas en este studio muestran un reflejo de la configuración de la movilidad científica mexicana durante su formación y de la migración científica hacia México. Esta configuración confirma que los centros de atracción para la formación científica se encuentran principalmente en países industrializados. Las 
cartografías, además de ser una representación de las relaciones científicas entre México y los demás países, reflejan igualmente los desplazamientos de prácticas y saberes científicos y tecnológicos, esto ya que los jóvenes investigadores al formarse en laboratorios y centros de investigación traen consigo métodos, técnicas y marcos teóricos que reproducen en su inserción en las entidades académicas y científicas en México.

Las asimetrías mostradas en los resultados de este estudio requieren ser estudiadas a profundidad, para lo cual es necesario efectuar otros estudios, tanto cuantitativos como cualitativos, ya que las cartografías expuestas en este documento solo nos permiten obtener un panorama sobre la movilidad y migración científicas.

Finalmente, las cartografías expuestas en este texto son complementarias, ya que en su conjunto muestran una geografía de las relaciones Norte-Sur y Sur-Sur en la ciencia y la tecnología a partir de la movilidad durante la formación de los científicos en México y la migración hacia este país. Sin embargo, detrás de estas movilidades, igualmente hay historias de vida, creaciones de instituciones, hechos históricos nacionales e internacionales y políticas públicas en diferentes niveles. Todos estos son elementos a tomar en cuenta para entender mejor estos fenómenos sociales que han contribuido a la formación y consolidación del actual sistema científico y tecnológico en México.

\section{Agradecimientos}

Esta investigación fue apoyada por el proyecto IA300916 del Programa de Apoyo a Proyectos de Investigación e Innovación Tecnológica (PAPIIT) de la UNAM. Además, el autor agradece a Gribran Barrera A., Técnico Académico del IIMAS, por su apoyo para la realización de las cartografías. 


\section{Referencias}

Ackers, L. (2005). Moving people and knowledge: Scientific mobility in the European Union. International Migration, 43(5), 99-131. https://doi.org/10.1111/j.14682435.2005.00343.x

Bornmann, L., Leydesdorff, L., Walch-solimena, C. y Ettl, C. (2011). Mapping excellence in the geography of science: An approach based on Scopus data. Journal of Informetrics, 5(4), 537-546. https://doi.org/10.1016/j.joi.2011.05.005

Contreras-Gómez, L. E., Baquero-Parra, R., Robles-Belmont, E. y Pérez-Angón, M. Á. (2015). Patrones de movilidad de los físicos mexicanos en el Sistema Nacional de Investigadores. Interciencia, 4O(8), 525-532.

de Haas, H. (2010). Migration and Development: International Migration Review, 44(1), 227-264. https://doi.org/10.1111/j.1747-7379.2009.00804.x

Delgado-Wise, R. y Chávez-Elorza, M. (2016). Migración calificada: entre la pérdida de talento y la oportunidad de transformar a México con innovación. Revista Mexicana de Política Exterior, 107(September), 117-135.

Delgado-Wise, R., Márquez-Covarrubias, H. y Rodríguez-Ramírez, H. (2009). Seis tesis para desmitificar el nexo entre migración y desarrollo. Migración y Desarrollo, (12), 27-52.

Didou, S. (2009). ¿Pérdida de cerebros y ganancia de saberes?: la movilidad internacional de recursos humanos altamente calificados en América latina y el Caribe. In S. Didou \& E. Gérard (Eds.), Fuga de cerebros, movilidad académica, redes científicas. Perspectivas latinoamericanas (pp. 25-62). Mexico: IESALC CINVESTAV - IRD.

Didou, S. y Gérard, E. (2009). Fuga de cerebros, movilidad académica y redes científicas. Perspectivas latinoamericanas. (S. Didou \& E. Gérard, Eds.). Mexico: IESALC - CINVESTAV - IRD.

Didou, S. y Gérard, E. (2010). El Sistema Nacional de Investigadores, veinticinco años después. La comunidad científica, entre distinción e internacionalización. ANUIES.

Docquier, F., Lohest, O. y Marfouk, A. (2005). Brain Drain in Developing Regions (1990-200o) (No. 1668). Bonn. Retrieved from https://papers.ssrn.com/sol3/papers.cfm?abstract_id=761624

Docquier, F. y Machado, J. (2016). Global Competition for Attracting Talents and the World Economy. The World Economy (Vol. 39). https://doi.org/10.1111/twec.12267

Findlay, A. M., King, R., Smith, F. M., Geddes, A. y Skeldon, R. (2012). World class? An investigation of globalisation, difference and international student mobility. 
Transactions of the Institute of British Geographers, 37(1), 118-131. https://doi.org/10.1111/j.1475-5661.2011.00454.x

Freitas, A., Levatino, A., y Pécoud, A. (2012). Introduction: New Perspectives on Skilled Migration. Diversities, 14(1), 1-7.

Frenken, K., Hardeman, S., y Hoekman, J. (2009). Spatial scientometrics: Towards a cumulative research program. Journal of Informetrics, 3(3), 222-232. https://doi.org/10.1016/j.joi.2009.03.005

García Pires, A. (2015). Brain Drain and Brain Waste. Journal of Economic Development, 4O(1), 1-34. https://doi.org/10.35866/caujed.2015.40.1.001

Gérard, E., y Kuri, R. G. (2009). ¿Endogamia o exogamia científica? La formación en el extranjero, una fuerte influencia en las prácticas y redes científicas, en particular en las ciencias duras. In S. D. Aupetit y E. Gérard (Eds.), Fuga de cerebros, movilidad académica, redes científicas. Perspectivas latinoamericanas (Primera, pp. 137-160). Ciudad de México: CINVESTAV.

Gérard, E., y Maldonado, E. (2009). "Polos de saber" y “cadenas de saber". Impactos de la movilidad estudiantil en la estructuración del campo científico mexicano. Revista de La Educación Superior, 38(152), 49-62. Retrieved from http://www.scielo.org.mx/scielo.php?script=sci_arttext\&pid=So18527602009000400004\&lng=es\&nrm=iso\&tlng=es

González Ramos, A. M. (2011). Atrayendo Talento: Estrategias de Movilidad de los profesionales altamente cualificados en España. Sociología y Tecnociencia, 2(1), $72-87$.

Jöns, H., Livingstone, D. N., y Meusburger, P. (2010). Interdisciplinary Geographies of Science. In H. Peter Meusburger; David N. Livingstone; Jöns (Ed.), Geographies of Science (pp. ix-xvii). Springer Netherlands. https://doi.org/10.1007/978-90481-8611-2

King, R. (2012). Geography and Migration Studies: Retrospect and Prospect. Population, Space and Place, 18(2), 134-153. https://doi.org/10.1002/psp.685

King, R., y Raghuram, P. (2013). International Student Migration : Mapping the Field and New Research Agendas. Population, Space and Place, 19(2), 127-137.

Kreimer, P. (2006). ¿Dependiente o integrados? La ciencia latinoamericana y la nueva división internacional del trabajo. NOMADAS, (24), 199-212.

Kreimer, P., y Zabala, J. P. (2006). ¿Qué conocimiento y para quién? Problemas sociales, producción y uso social de conocimientos científicos sobre la enfermedad de chagas en argentina. REDES, 12(023), 49-78.

Leydesdorff, L., y Persson, O. (2010). Mapping the geography of science: Distribution patterns and networks of relations among cities and institutes. Journal of the 
American Society for Information Science and Technology, 61(8), 1622-1634. https://doi.org/10.1002/asi.21347

Livingstone, D. N. (2003). Putting Science in its Place. Geographies of Scientific Knowledge (Reimpresa). The University Chicago Press.

Luna-Ochoa, S. M. A., Robles-Belmont, E., y Suaste-Gomez, E. (2016). A profile of Mexico's technological agglomerations: The case of the aerospace and nanotechnology industry in Quer??taro and Monterrey. Technology in Society, 46, $120-125$.

Mahroum, S. (2000). Scientific Mobility. An Agent of Scientifique Expansion and Institutional Empowerment. Science Communication, 21(4), 367-378.

Mattoo, A., Neagu, I. C., y Özden, Ç. (2005). Wps3581 (No. WPS3581). Washintong, DC.

Meusburger, P. Livingstone, D. N. y Jöns, H. (2010). Geographies of Science. (H. Peter Meusburger; David N. Livingstone; H. Jöns, Eds.). Springer Netherlands. https://doi.org/10.1007/978-90-481-8611-2

Mayer, L. (2015). Rutas de incertidumbre. Ideas alternativas sobre la génesis de la probabilidad, siglos XVI y XVII. México City: Fondo de Cultura Económica.

Meyer, J., y Brown, M. (1999). Scientific Diasporas : A New Approach to the Brain Drain by. Management of Social Transformations.

Meyer, J., Kaplan, D., y Charum, J. (2001). Nomadisme des scientifiques et nouvelle géopolitique du savoir. Revue Internationale Des Sciences Sociales, (168), 341354 .

Miller, J. H., y Page, S. E. (2007). Complex Adaptive Systems: An Introduction to Computational Models of Social Life. Tetrahedron Organic Chemistry Series (Vol. 27). https://doi.org/10.1016/S1460-1567(08)10011-3

Özden, C. (2006). Brain Drain in Latin America (UN/POP/EGM-MIG/2005/10). Mexico.

Pellegrino, A. (2001). Éxodo, movilidad y circulación: nuevas modalidades de la migración calificada. Notas de Población, 28(73), 129-162.

Quet, M. (2014). La circulation des savoirs. Interdisciplinarité, concepts nomades, analogies, métaphores. Frédéric DARBELLAY (éd.), Berne, Peter Lang, 2012. Revue d'anthropologie Des Connaissances, 8(1), 221-224. https://doi.org/10.3917/rac.022.0221

Raghuram, P. (2013). Theorising the Spaces of Student Migration. Population, Space and Place, 19(2), 138-154. https://doi.org/10.1002/psp.1747

Robles-Belmont, E. (2009). Las redes científicas como respuesta a la emergencia de las nanociencias y nanotecnologías. REDES, 15(29), 93-111. 
Robles-Belmont, E. (2014). Cooperación científica internacional en la nanomedicina de México. Observatorio Del Desarrollo, 3(12), 29-33.

Robles-Belmont, E., Ruiz-León, A., Aranda-Román, J. A., y Galarza-Barrios, P. (2013). ¿Cómo Clasificar la Ciencia y la Tecnología? Algunas Propuestas a partir de las clasificaciones de la UNESCO y la OCDE para el Caso de México Introducción. In Congreso ALTEC 2013: gestión de ciencia e tecnología en el contexto LatinoIberoamericano (pp. 1-13).

Straubhaar, T. (2000). International Mobility of Highly Skilled: Brain Gain, Brain Drain or Brain Exchange (HWWA). The International Mobility of Talent: Types, Causes, and Development Impact.

Tejada, G. (2012). Mobility, knowledge and cooperation: scientific diasporas as agents of development migration and development. Migration and Development, 10(18), 59-92. Retrieved from https://www.researchgate.net/profile/Gabriela_Tejada2/publication/283742038 _Mobility_Knowledge_and_Cooperation_Scientific_Diasporas_as_Agents_of_C hange/links/57049eb8o8ae44d70ee065f2/Mobility-Knowledge-and-CooperationScientific-Diasporas-as-Agents-of-Cha

Valdez-Lafarga, C., y León-Balderrama, J. I. (2015). Hacia una taxonomía de los sistemas regionales de innovación en México Towards a taxonomy of regional innovation systems in Mexico. Economía, Sociedad y Territorio, XV(48), 517553.

Vessuri, H. (2003). La Ciencia y la Educación Superior en el Proceso de Internacionalización. Elementos de un Marco Conceptual para América Latina. UNESCO Forum Occasional Paper Series (Vol. 3/S).

Ziman, J. M. (1977). The International Scientific Community. Minerva, 15(1), 83-93. 